Abstract

\title{
Comparing Different Larval Food Sources and Temperature Regimes for the Rearing of Culicoides obsoletus/scoticus Complex Midges, the Predominant Bluetongue, and Schmallenberg Virus Vectors in Northern Europe ${ }^{+}$
}

\author{
Charlotte Sohier *, Severine Matthijs and Nick De Regge \\ Unit of Enzootic, Vector-Borne and Bee Diseases, Sciensano, 1180 Brussels, Belgium; \\ Severine.Matthijs@sciensano.be (S.M.); Nick.Deregge@sciensano.be (N.D.R.) \\ * Correspondence: Charlotte.Sohier@sciensano.be \\ † Presented at Viruses 2020-Novel Concepts in Virology, Barcelona, Spain, 5-7 February 2020. \\ Published: 17 June 2020
}

\begin{abstract}
During the last decade, Europe was confronted with the emergence of two Culicoidesborne viruses, bluetongue virus and Schmallenberg virus. Both diseases caused economic losses in cattle, sheep, and goats. Despite their importance, only limited knowledge is available on the developmental stages of Culicoides obsoletus complex midges, and a lab-reared colony has not yet been established. Therefore, this experimental study aims to compare the emergence patterns from field-collected C. obsoletus complex from egg to adult that were exposed to different combinations of temperature and larval substrates (T1: $1 \%$ liquid broth (LB) agar at $24{ }^{\circ} \mathrm{C} ; \mathrm{T} 2: 1 \%$ LB agar with dung patches at $24{ }^{\circ} \mathrm{C}$; T3: $1 \%$ LB agar at $28{ }^{\circ} \mathrm{C}$ ). The rearing dishes were kept in an environmental chamber under an $8 \mathrm{~h} / 16 \mathrm{~h}$ dark-light regime and $80 \%-85 \%$ humidity. Algae and nematodes were provided as a food source. The average period required to develop from egg to adult was 24 days and varied strongly in each of the conditions (T1: 15-47 days; T2: 14-35 days; T3: 20-37 days). A bias of sex ratio was observed towards the production of males in all three treatments with only $23.9 \%$ (T1), 23.5\% (T2), and 0\% (T3) females. Larval mortality was highest in T3 (25.9\%), followed by T2 $(8.6 \%)$, and $\mathrm{T} 1(1.8 \%)$. All pupae produced adults within 1 to 10 days, and the emergence rates for pupae varied strongly between treatments: $49.5 \%$ (T1), $71.6 \%$ (T2), and 38.5\% (T3). This study shows that the $C$. obsoletus complex can be reared under laboratory conditions from blood-fed, wild-caught females to emerged progeny. More larval substrates and food sources should be tested with the aim of obtaining a 1:1 sex ratio to bring us one step closer to a viable lab-reared colony.
\end{abstract}

Keywords: Culicoides obsoletus s.l.; development lifecycle; oviposition; vector; emergence; arbovirus; artificial blood feeding

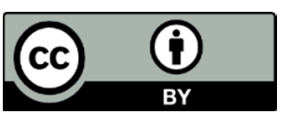

(C) 2020 by the authors. Licensee MDPI, Basel, Switzerland. This article is an open access article distributed under the terms and conditions of the Creative Commons Attribution (CC BY) license (http://creativecommons.org/licenses/by/4.0/). 